\title{
The SFR Efficiency of HI Gas in the Outskirts of Star Forming Galaxies
}

\author{
Marc Rafelski \\ NASA Postdoctoral Fellow at Goddard Space Flight Center \\ Code 665, Greenbelt, MD 20771, USA \\ marc.a.rafelski@nasa.gov
}

\begin{abstract}
In order to understand the origin of the decreased star formation rate (SFR) efficiency of neutral atomic hydrogen gas measured in Damped Ly $\alpha$ Systems (DLAs) at $z \sim 3$, we measure the SFR efficiency of atomic gas at $z \sim 1, z \sim 2$, and $z \sim 3$ around star-forming galaxies. We create galaxy stacks in these three redshift bins, and measure the SFR efficiency by combining DLA absorber statistics with the observed rest-frame UV emission in the galaxies' outskirts. We find that the SFR efficiency of HI gas is $\sim 3 \%$ of that predicted by the KS relation. We find no significant evolution in the SFR efficiency with redshift, although simulations and models predict a decreasing SFR efficiency with decreasing metallicity and thus with increasing redshift. We discuss possible explanations for this decreased efficiency without an evolution with redshift.
\end{abstract}

Keywords. galaxies: high-redshift, galaxies: evolution, (galaxies:) intergalactic medium, (galaxies:) quasars: absorption lines.

\section{Introduction}

Damped Ly $\alpha$ systems (DLAs) trace most of the neutral Hi gas in the Universe and are typically studied in absorption to background quasars (QSOs). In this paper we use a statistical comparison to connect absorption line studies tracing the gas, with starforming galaxies (SFGs) measured in emission. We compare observed emission, which likely originates from DLAs, to that predicted by combining the column-density distribution function of HI gas, $f\left(N_{\mathrm{HI}}\right)$, at high redshift with the Kennicutt-Schmidt (KS) relation (Kennicutt et al. 1998) and a geometrical model (Rafelski et al. 2011).

The star formation rate (SFR) efficiency, defined here as the fractional decrease in the normalization of the KS relation, was previously determined to be $<10 \%$ at $z \sim 3$ in isolated regions by Wolfe \& Chen (2006) and in the outskirts of SFGs by Rafelski et al. (2011). There are multiple possible effects contributing to the lower SFR efficiencies than predicted by the KS relation, such as the lower metallicity of the HI gas, a higher background radiation field at high redshift, and the role of molecular versus atomic hydrogen gas in star formation. Measurements of the evolution of the SFR efficiency and metallicity over time help distinguish between these different possibilities, and here we determine the SFR efficiency at $z \sim 1$ and $z \sim 2$, while also improving it at $z \sim 3$.

\section{Analysis}

We measure the rest-frame FUV emission in the outskirts of SFGs selected from the photometric redshift catalog by Rafelski et al. (2015). For each redshift $(z \sim 1, z \sim 2$, and $z \sim 3)$ we create a composite stack and extract radial surface brightness profiles, which show spatially extended star formation in the SFG outskirts. We use the radial surface brightness profiles of the SFGs to measure the evolution in the SFR efficiency from $z \sim 1$ to $z \sim 3$ by comparing the observed emission to that expected from absorption statistics of the HI gas, described by $f\left(N_{\mathrm{HI}}\right)$. While this is well determined at $z>2$, it 


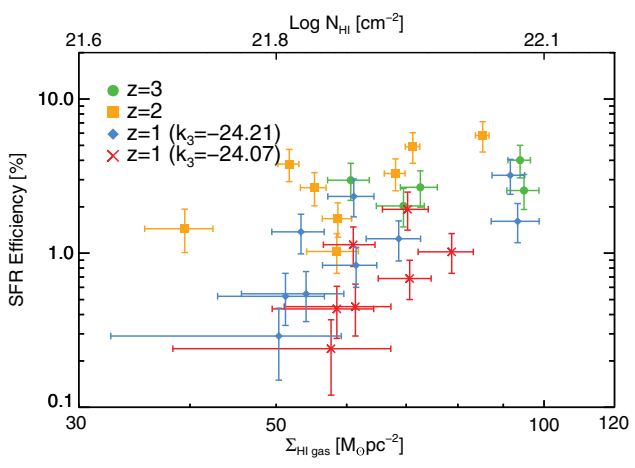

Figure 1. SFR efficiency versus implied $\Sigma_{\text {gas }}$ shown for different redshifts. The green circles correspond to the $z \sim 3$ SFGs, the orange squares to those at $z \sim 2$, and the blue diamonds and red crosses are for those at $z \sim 1$. The data show no evolution with redshift.

is very uncertain at lower redshifts (Neeleman et al. 2016). We fit the $f\left(N_{\mathrm{HI}}\right)$ data from Noterdaeme et al. (2012) with a double power law, and then measure the evolution of the normalization of $f\left(N_{\mathrm{HI}}\right), k_{3}$, as a function of redshift. Due to the uncertainty at $z<2$, we consider two possible values of $k_{3}$ at $z \sim 1$.

We determine the SFR efficiency of atomic-dominated neutral hydrogen gas at each redshift by comparing the emission observed in the outskirts of SFGs to the emission expected from DLAs based on a model which predicts the SFR density per intensity interval around the SFGs (see Rafelski et al. (2011) and Rafelski et al. (2016)). The resultant SFR efficiency ranges from $\sim 1-6 \%$ depending on the surface brightness (and therefore the column density, $N_{\mathrm{HI}}$ ), with a mean value around $2 \%$. We investigate any potential evolution in the SFR efficiency with redshift in Fig. 1, which shows the SFR efficiency as a function of $\Sigma_{\text {gas }}$ for each redshift bin. We do not observe any clear evolution in the SFR efficiency with redshift, regardless of the choice of $k_{3}$ for the $z \sim 1$ point. Further details of the analysis are provided in Rafelski et al. (2016).

\section{Discussion}

Cosmological galaxy simulations find that the primary source for lower SFR efficiencies at high redshift is a decrease in the dust-content. The low dust content of DLAs, as traced by the metallicity, could therefore explain the reduced SFR efficiencies. We compare our results to the simulations by Gnedin \& Kravtsov (2010), which include a metallicity dependent model of molecular hydrogen, at $z \sim 3$ for gas with metallicity below $0.1 Z_{\odot}$. Figure 2 (left) shows that the simulations predict the same SFR efficiency as measured here. Gnedin \& Kravtsov (2010) conclude that the lower metallicity, and therefore lower dust-to-gas ratio, causes a decrease in the amplitude of the KS relation.

We also investigate the reduced efficiencies with analytic models. The Krumholz (2013) model explicitly computes the behavior of HI-dominated gas in the galaxy outskirts, and provides us with the $\Sigma_{\text {SFR }}$ as a function of the metallicity and $\Sigma_{\text {gas }}$. The model reproduces the observed low efficiencies at the metallicities of DLAs. The model can also be used to predict the expected $\Sigma_{\mathrm{SFR}}$ as a function of $\Sigma_{\text {gas }}$ at different metallicities, and therefore redshifts. The metallicity of DLA gas increases with decreasing redshift (Rafelski et al. 2012 ), which translates to a change of $\sim 0.5$ dex in the $\Sigma_{\mathrm{SFR}}$, as shown in Figure 2 (left). However, we find no evolution in the SFR efficiency of the Hi gas.

An alternative explanation of the reduced SFR efficiency is the role of molecular versus atomic hydrogen gas in star formation. An analysis of the atomic-dominated gas in the outskirts of local spiral and dwarf galaxies also finds a reduced SFR efficiency (Bigiel et al. 2010, Elmegreen et al. 2015). Figure 2 (right) compares the SFR efficiency measured in DLA gas in the outskirts of SFGs to that of the HI gas in the outskirts of local spiral and dwarf galaxies. These measurements at $z=0$ show SFR efficiencies similar to 

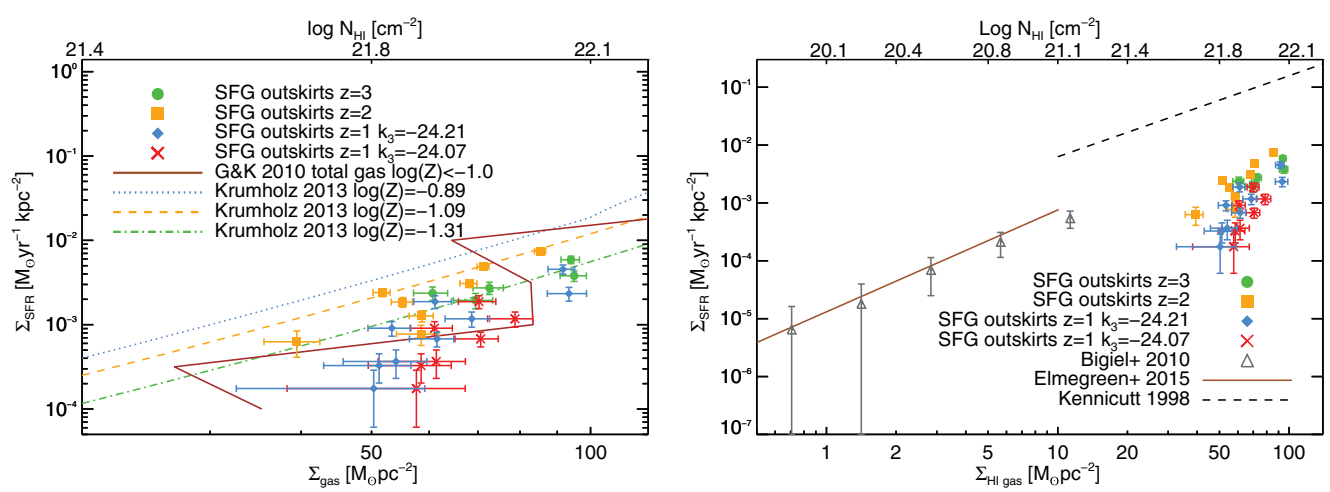

Figure 2. Left: Comparison of the SFR efficiency of DLA gas in the outskirts of SFGs at $z \sim 1-3$ with the $z \sim 3$ simulation by Gnedin \& Kravtsov (2010) and the analytic KMT+ model by Krumholz (2013). The KMT+ model predictions are for the metallicities of DLAs at the redshifts of the binned SFGs, with the colors matching those of the corresponding measurements at each redshift: green for $z=3$, orange for $z=2$, and blue for $z=1$. The model provides a measure of the expected evolution in the SFR as a function of redshift due to the metallicity evolution of DLAs, which would evolve from the green line at $z=3$ to the blue line at $z=1$. The data are broadly in agreement with the simulations and models, although the data do not reproduce the predicted metallicity evolution. Right: Comparison of the SFR efficiency of DLA gas in the outskirts of SFGs at $z \sim 1-3$ with local measurements. This shows that the upper limits from direct DLA observations are consistent with both our measurements and the local HI gas.

those presented here at $z \sim 1-3$, suggesting that the efficiency of this gas is not changing significantly over time or strongly with metallicity.

The similar efficiency of HI-dominated gas at both low and high redshift and at different metallicities, combined with the lack of any observed evolution in the efficiency of DLAs as a function of redshift (and therefore metallicity), suggests that the reduced SFR efficiency is likely driven by the low molecular content of the atomic-dominated phase. At the same time, the metallicity could play a secondary effect on the efficiency by regulating the conversion between atomic and molecular gas. Theory predicts that there is a metallicity dependence on this transition, which would explain the observed high column-densities of DLAs without much molecular gas. In this scenario, the metallicity of the DLA gas is too low for the atomic gas to form significant amounts of molecular gas at the given surface densities. This may explain how the KMT + model does not reproduce the lack of evolution in the efficiency of the DLA gas, while at the same time reproducing the SFR efficiency of the local Hi gas (Krumholz 2013).

\section{References}

Bigiel, F., et al. 2010b, AJ, 140, 1194

Elmegreen, B. G. \& Hunter, D. A. 2015, ApJ, 805, 145

Gnedin, N. Y. \& Kravtsov, A. V. 2010, ApJ, 714, 287

Kennicutt, R. C. 1998a, ARA\&A, 36, 189

Krumholz, M. R. 2013, MNRAS, 436, 2747

Neeleman, M., et al. 2016, ApJ, 818, 113

Noterdaeme, P., et al. 2012, A\& A, 547, L1

Rafelski, M., et al. 2016, accepted for publication in ApJ

Rafelski, M., et al. 2015, AJ, 150, 31

Rafelski, M., et al. 2012, ApJ, 755, 89

Rafelski, M., Wolfe, A. M., \& Chen, H.-W. 2011, ApJ, 736, 48

Wolfe, A. M. \& Chen, H.-W. 2006, ApJ, 652, 981 